\title{
Guaranteed and prospective Galactic TeV neutrino sources
}

\author{
Matthew D. Kistler ${ }^{1, *}$ and John F. Beacom ${ }^{1,2, \dagger}$ \\ ${ }^{1}$ Department of Physics, The Ohio State University, Columbus, Ohio 43210, USA \\ ${ }^{2}$ Department of Astronomy, The Ohio State University, Columbus, Ohio 43210, USA
}

(Received 5 July 2006; published 18 September 2006)

\begin{abstract}
Recent observations, particularly from the HESS Collaboration, have revealed rich Galactic populations of TeV gamma-ray sources, including a collection unseen in other wavelengths. Many of these gamma-ray spectra are well measured up to $\sim 10 \mathrm{TeV}$, where low statistics make observations by air Cerenkov telescopes difficult. To understand these mysterious sources, especially at much higher energies - where a cutoff should eventually appear-new techniques are needed. We point out the following: (1) For a number of sources, it is very likely that pions, and hence TeV neutrinos, are produced; (2) As a general point, neutrinos should be a better probe of the highest energies than gamma rays, due to increasing detector efficiency; and (3) For several specific sources, the detection prospects for $\mathrm{km}^{3}$ neutrino telescopes are very good, $\sim 1-10$ events/year, with low atmospheric neutrino background rates above reasonable energy thresholds. Such signal rates, as small as they may seem, will allow neutrino telescopes to powerfully discriminate between models for the Galactic TeV sources, with important consequences for our understanding of cosmic-ray production.
\end{abstract}

\section{INTRODUCTION}

The field of $\mathrm{TeV}$ gamma-ray astronomy is exploring energy regimes that have been, until recently, out of reach to astrophysicists. Yet, even as the catalog of $\mathrm{TeV}$ sources continues to grow, it is still debated whether the observed gamma rays are produced leptonically, through the inverse Compton scattering of energetic electrons on ambient photons $\left(e^{-} \gamma \rightarrow \gamma e^{-}\right)$, or hadronically, through neutral pion decay $\left(\pi^{0} \rightarrow \gamma \gamma\right)$. Air Čerenkov telescopes, such as HESS, can measure a source spectrum with high precision for $E_{\gamma} \sim 1-10 \mathrm{TeV}$ [1]. One might hope that as we probe higher energy gamma rays, indications of their true origin would be revealed. However, at energies $\gtrsim 10 \mathrm{TeV}$, the difficulties of gamma-ray astronomy become more pronounced, due to the low statistics of the quickly declining signal spectra (see Fig. 1).

It is well established that a distinctive feature of a pionic (hadronically produced) gamma-ray spectrum is an accompanying flux of neutrinos [2-5]. These neutrinos originate from the decay of charged pions $\left(\pi^{+}, \pi^{-}\right)$, which are produced in approximately equal numbers with neutral pions in proton-proton scattering. Relative to gamma-ray telescopes, the new $\mathrm{km}^{3}$ neutrino telescopes $[6,7]$ will have several advantages that result in improved performance at the highest energies. The rapidly falling atmospheric neutrino background, rising neutrino-nucleon cross section $\left(\sigma_{\nu N} \sim E_{\nu}\right)$, and increasing muon range, which effectively expands the (already large) detector volume $\left(R_{\mu} \sim \ln E_{\mu}\right)$, all help to amplify the diminished flux at these energies. In fact, as the background quickly becomes negligible in the $\mathrm{TeV}$ range, the detection of any high energy neutrinos from

\footnotetext{
*Electronic address: kistler@mps.ohio-state.edu

†Electronic address: beacom@mps.ohio-state.edu
}

a source could significantly indicate a hadronic production mechanism.

Neutrino telescopes have capabilities far beyond breaking the degeneracy between leptonic and hadronic production models. Spectral features in the highest energy regime,

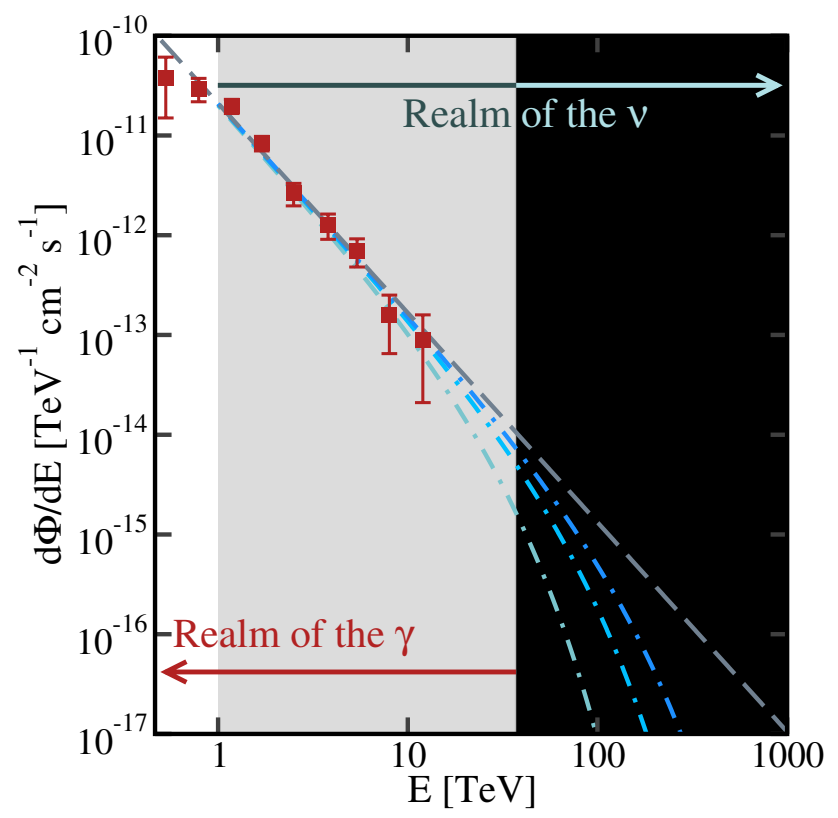

FIG. 1 (color online). Ranges over which gamma-ray and neutrino observations can measure a source spectrum $\left(E_{\gamma} \simeq\right.$ $\left.E_{\pi} / 2, E_{\nu} \simeq E_{\pi} / 4\right)$. Squares are HESS measurements of Vela Jr., fit by a power law (dashed line). Neutrino telescopes can probe higher energies to distinguish between pionic spectra with cutoffs (dot-dashed lines) by measuring the $\nu_{\mu}$-induced muon and shower spectra. Muon rates of $N\left(E_{\mu}>1 \mathrm{TeV}\right) \sim 3-6 \mathrm{yr}^{-1}$ correspond to the dot-dashed lines (see Sec. IV), illustrating the sensitivity of neutrino observations. 
especially an expected cutoff (related to the maximum accelerated proton energies), shall not remain inaccessible to observation. Measurement of the energies of neutrinoinduced muons and showers, which are related to the original charged pion energy, can probe the source proton spectrum in a complementary manner to gamma-ray observations, which effectively measure the neutral pion spectrum [8,9]. The sensitivities of these two independent approaches, including the regime where they coincide, are illustrated in Fig. 1. The ability to accurately measure neutrino-induced muon spectra greatly improves the prospects for detecting point sources, as the harder source spectra dominate the atmospheric background above $\sim 1 \mathrm{TeV}$. While muon tracks have better angular resolution ( $\lesssim 1^{\circ}$ ), neutrino showers $\left(\sim 10^{\circ}\right.$ in water) more faithfully trace the spectrum. Shower observations, which measure the $\nu_{e}$ and $\nu_{\tau}$ fluxes, when combined with muon data, also allow for the study of the ratio of neutrino flavors arriving from a source $[10,11]$.

Considering the latest observations of Galactic $\mathrm{TeV}$ sources, we calculate the corresponding spectra of detectable neutrino-induced muons and showers, assuming only that the observed gamma-ray spectra are pionic, for a range of possible high energy cutoffs in the spectra. Relative to analyses in which the total numbers of signal and background events are counted (e.g., Ref. [12]), a maximum likelihood analysis would have much more power. For example, below $\sim 1 \mathrm{TeV}$, a single event has a much greater probability of being the background than at $10 \mathrm{TeV}$, where the source signal is dominant. Spectra allow for such an approach, which takes full advantage of experimental data, in studying the high energy behavior of $\mathrm{TeV}$ sources. Resolving hadronic activity at these extreme energies would provide clear evidence concerning the sources of Galactic cosmic rays [13].

Atmospheric cosmic-ray showers give rise to high rates of down-going muons, forcing a neutrino telescope to search for up-going muons resulting from neutrino interactions. IceCube is well situated to utilize the high resolution of these $\nu_{\mu}$-induced muons in observing northern-sky sources. However, a detector is needed in the northern hemisphere to accurately locate southern-sky neutrino sources, although IceCube may also measure shower rates from particularly bright sources. Together, IceCube and a $\mathrm{km}^{3}$ Mediterranean detector will provide full-time coverage of the entire sky, a feature distinct to neutrino telescopes. The combined observations from these detectors can be used to study compound objects, like the Vela complex, by: (1) Discovering neutrino sources through high-resolution $\nu_{\mu}$-induced muons; (2) Confirming agreement with gamma-ray observations in the low energy regime; (3) Examining previously unexplored energies using muons and showers together.

The sources that we examine in detail are briefly discussed in Sec. II. Our empirical calculations, based upon observed $\mathrm{TeV}$ spectra, should be compared with previous Galactic neutrino studies, which have considered theoretical production models or less recent data (e.g. Refs. [1425]). In two very recent works, by Lipari [8] and Kappes, Hinton, Stegmann, and Aharonian [9], a similar approach to ours has been followed, with complementary perspectives and details.

\section{PROMISING TEV NEUTRINO SOURCES}

Vela Region-Of particular interest amongst prospective neutrino sources, the shell-type supernova remnant (SNR) Vela Jr. (RX J0852.2-4622) is one of the brightest objects in the southern TeV sky. The hard, intense $\mathrm{TeV}$ gamma-ray spectrum of Vela Jr., best explained as being pionic in nature, makes it an intriguing object to study [26]. Additionally, the morphology of the $\mathrm{TeV}$ emission may make this one of the most interesting astrophysical neutrino sources. HESS has also measured a TeV spectrum from Vela $\mathrm{X}$, the pulsar wind nebula (PWN) associated with the larger Vela SNR, and advanced a leptonic origin [27]. However, if this spectrum is instead pionic, as proposed in Ref. [28], the accompanying neutrino flux would be easily detectable. The nondetection of neutrino events from such an intense source would allow for a significant test of leptonic production in a short period of observation.

Galactic Center Diffuse Emission-HESS has also recently discovered a region of diffuse $\mathrm{TeV}$ emission from the Galactic Center ridge [29]. This source has several interesting features which merit further investigation. The large extent of the emission, hardness of the spectrum, high gas density (which is well correlated with the $\mathrm{TeV}$ emission), and strong magnetic fields in the region leave very little doubt that this spectrum is pionic [29]. In addition, the total flux from this region is actually about twice as intense as that of the previously discovered source coincident with Sgr A* [30]. Measurement of the accompanying neutrino flux would provide an independent confirmation of the means of production, something which has never been possible.

Unknown Knowns and Known Unknowns-We also examine the neutrino detection prospects of other known TeV sources, including SNR RX J1713-3946, the spectrum of which has been measured twice by HESS and determined to likely be pionic [31,32]. The calculated $\nu_{\mu}$-induced muon fluxes based upon the two different sets of HESS data illustrate the effects of different high energy spectral assumptions. Recently, HESS has reported the discovery of $4 \mathrm{TeV}$ sources in the Galactic plane which have no apparent counterparts at other wavelengths [33,34]. In addition to these, EGRET previously discovered numerous unidentified sources of $\mathrm{GeV}$ gamma rays in the Milky Way [35]. The confirmed observation of neutrinos from these sources would also reveal an intimate connection between $\mathrm{TeV}$ gamma rays and cosmic rays. 
Furthermore, the understanding of these and other gammaray sources may account for the $\mathrm{TeV}$ excess [36].

\section{BASICS OF NEUTRINO DETECTION}

In high energy $p$ - $p$ scattering, $\pi^{+}, \pi^{-}$, and $\pi^{0}$ are produced in nearly equal numbers [37]. Gamma rays are the result of the decay $\pi^{0} \rightarrow \gamma \gamma$, while neutrinos originate from the $\pi^{+} \rightarrow \mu^{+} \nu_{\mu} \rightarrow e^{+} \bar{\nu}_{\mu} \nu_{e} \nu_{\mu}$ and $\pi^{-} \rightarrow \mu^{-} \bar{\nu}_{\mu} \rightarrow$ $e^{-} \nu_{\mu} \bar{\nu}_{e} \bar{\nu}_{\mu}$ decay channels. The ratio of neutrinos to photons from pion decay is easily found. From charged pion decay, the resulting initial neutrino flavor ratio, $\nu_{e}: \nu_{\mu}: \nu_{\tau}$, is 1:2:0. During the traversal of astrophysical distances, vacuum neutrino oscillations transform this ratio to $1: 1: 1$. In neutrino telescopes, neutrinos and antineutrinos are practically indistinguishable. We can then consider their sum, $\nu+\bar{\nu}$, and average the cross section in all calculations. All further references to neutrinos will imply $\nu+\bar{\nu}$. Thus, for equal pion multiplicities, each photon from $\pi^{0}$ decay corresponds to one neutrino of each flavor $\left(N_{\gamma}=\right.$ $N_{\nu_{e}}=N_{\nu_{\mu}}=N_{\nu_{\tau}}=N_{\nu}$ ). The typical energy of the neutrinos resulting from these decays is $\sim 1 / 2$ of the gammaray energy from $\pi^{0}$ decay. The resulting $\nu+\bar{\nu}$ spectrum is then shifted, relative to the source gamma-ray spectrum of $d \Phi_{\gamma} / d E_{\gamma}=\phi_{\gamma} E_{\gamma}^{-\Gamma}$, as

$$
\frac{d \Phi_{\nu}}{d E_{\nu}}=\left(\frac{1}{2}\right)^{\Gamma-1} \phi_{\gamma} E_{\nu}^{-\Gamma}=\phi_{\nu} E_{\nu}^{-\Gamma},
$$

where we consider each neutrino flavor separately.

We will generally refer to the normalization of the gamma-ray spectrum as $\phi_{\gamma}$, the differential photon flux at $1 \mathrm{TeV}$ (in $\mathrm{TeV}^{-1} \mathrm{~cm}^{-2} \mathrm{~s}^{-1}$ ), which is commonly used in gamma-ray astronomy. Typical source gamma-ray spectra have $\phi_{\gamma} \sim 5-20 \times 10^{-12} \mathrm{TeV}^{-1} \mathrm{~cm}^{-2} \mathrm{~s}^{-1}$ and $\Gamma \sim$ 1.8-2.4. For greater detail on the relationship between the initial proton spectrum and the resulting product spectra, see Ref. [38]. Pions can also be produced, in a different ratio, in $p-\gamma$ scattering, but this is generally only important at much higher energies [39]. A neutrino flux may be observed through neutrino-induced muons and electromagnetic (and hadronic) cascades, which are referred to as showers. We shall consider both methods in further detail.

\section{A. Muon detection}

Our analysis of muon detection will be limited to $\nu_{\mu}$ charged-current (CC) events with an observable final energy of $E_{\mu}>0.5 \mathrm{TeV}$, which can be detected through their Cerenkov emission with an angular resolution of $\lesssim 1^{\circ}$ [40]. It is important to recognize that the relevant quantity in these events is the measured energy of the muon, which can be reconstructed from radiative losses in the detector [41-44]. As muons can be produced far outside of the detector, with energy loss prior to entering, results of calculations are given in terms of $E_{\mu}$ (at the detector) instead of $E_{\nu}$.

We can consider $\nu_{\mu}$-induced muons produced both inside and outside of the instrumented volume of the detector. Contained event rates can be found by combining the neutrino flux, detector mass, and $\sigma_{\mathrm{CC}}$ for neutrino-nucleon scattering. We use the average of the $\nu-N$ and $\bar{\nu}-N$ cross sections, as computed in Ref. [45], and $\left\langle y\left(E_{\nu}\right)\right\rangle$ from Ref. [46]. We consider a detector that is entirely composed of, and enclosed by, water (ice), with a $\mathrm{km}^{2}$ detector area, which is a reasonable approximation at these energies. Using an IceCube-like effective detector area, such as computed in Ref. [47], would lead to the lower energy muon spectrum being slightly suppressed. An actual $\mathrm{km}^{3}$ detector will be situated on top of solid rock, which will enhance the upward-going neutrino-induced muon rates relative to our calculations. Also, muons produced via $\nu_{\tau}$ CC interactions, which contribute to the total muon flux through the $\tau^{ \pm} \rightarrow \mu^{ \pm} \nu_{\mu} \nu_{\tau}$ decay channel, will not be considered [37,48,49]. Our formulas follow those of Gaisser [37,50], with appropriate approximations. Considering the uncertainties in both the gamma-ray spectra and neutrino telescope performance, which may not be fully understood until the actual detectors are calibrated, as well as the low statistics, our calculations are sufficient for the scope of this work.

Using these parameters, the spectrum of contained $\nu_{\mu}$-induced muon events is calculated as

$$
\left(\frac{d N_{\mu}}{d E_{\mu}}\right)_{\mathrm{cont}}=\kappa V_{\mathrm{det}} \frac{d \Phi_{\nu}}{d E_{\nu}} e^{-E_{\nu} / E_{\nu}^{\mathrm{cut}}} \sigma_{\mathrm{CC}}\left(E_{\nu}\right) e^{-\tau_{\oplus},}
$$

where $V_{\text {det }}$ is the detector volume, $E_{\nu}^{\text {cut }}$ is an assumed exponential cutoff in the neutrino spectrum, and the term $\kappa=N_{A} \rho T\left\langle 1-y\left(E_{\nu}\right)\right\rangle^{-1}$ takes into account observation time $(T)$, normalization of the muon spectrum, and the molar density of water. The energy of the produced muon is related to the original neutrino energy as $E_{\mu}=$ $\left\langle 1-y\left(E_{\nu}\right)\right\rangle E_{\nu}$. The term $e^{-\tau_{\oplus}}$, with $\tau_{\oplus}=N_{A} \lambda_{\oplus} \sigma_{\text {tot }}\left(E_{\nu}\right)$, accounts for neutrino attenuation due to scattering within Earth. Here, $\sigma_{\text {tot }}=\sigma_{\mathrm{CC}}+\sigma_{\mathrm{NC}}$ and $\lambda_{\oplus}$ is the average column depth (in cm.w.e.) of Earth based upon the declination of the source [46]. This attenuation factor only becomes important at $E_{\nu} \gtrsim 10 \mathrm{TeV}$, and varies with declination, as discussed in Ref. [8].

A high energy muon born outside of the detector may still be detectable when it enters the instrumented detector volume. To find the propagation range of a muon, we assume an average continuous muon energy loss of

$$
\frac{d E}{d X}=-\alpha-\beta E,
$$

where $\alpha=2.0 \times 10^{-6} \mathrm{TeV} \mathrm{cm}^{2} \mathrm{~g}^{-1}$ and $\beta=4.2 \times$ $10^{-6} \mathrm{~cm}^{2} \mathrm{~g}^{-1}[42,48]$. Integrating the average energy loss results in a range for a muon of initial energy $E_{\mu}$ of 


$$
R_{\mu}\left(E_{\mu}, E_{\mu}^{f}\right)=\frac{1}{\beta} \ln \left[\frac{\alpha+\beta E_{\mu}}{\alpha+\beta E_{\mu}^{f}}\right],
$$

where $E_{\mu}^{f}$ is the energy of the muon as it enters the detector. As the muon range (typically a few $\mathrm{km}$ ) increases with energy, so does the effective volume of the detector. The observed through-going spectrum, accounting for the probability of observing a muon entering the detector with energy $E_{\mu}$, can then be calculated as

$$
\begin{aligned}
\left(\frac{d N_{\mu}}{d E_{\mu}}\right)_{\mathrm{thru}}= & \frac{N_{A} \rho T A_{\mathrm{det}}}{\alpha+\beta E_{\mu}} \\
& \times \int_{E_{\mu}}^{\infty} d E_{\nu} \frac{d \Phi_{\nu}}{d E_{\nu}} e^{-E_{\nu} / E_{\nu}^{\text {cut }}} \sigma_{\mathrm{CC}}\left(E_{\nu}\right) e^{-\tau_{\oplus},}
\end{aligned}
$$

where $A_{\text {det }}$ is assumed to be $1 \mathrm{~km}^{2}$. It should be noted that our use of the total $\sigma_{\mathrm{CC}}$ and $\langle y\rangle$ may overestimate the muon signal rates by $\sim 20 \%-30 \%$ (perhaps more for spectra harder than $E^{-2}$ ). However, the atmospheric background will also be overestimated by a similar, even slightly larger amount.

\section{B. Shower detection}

For bright point sources or low surface brightness extended sources, shower events caused by $\nu_{e}, \nu_{\tau} \mathrm{CC}$ interactions can be used to effectively determine the source spectrum. To detect shower events, they must be at least partially enclosed within the instrumented volume. The shower signal is dominated by $\nu_{e}$ and $\nu_{\tau}$ CC events, which transfer $\sim 100 \%$ of the original neutrino energy to the shower $\left(E_{\mathrm{sh}} \approx E_{\nu}\right)$. The measurable shower spectrum, given in terms of the observed shower energy $E_{\mathrm{sh}}$,

$$
\left(\frac{d N_{\mathrm{sh}}}{d E_{\mathrm{sh}}}\right)_{\mathrm{CC}}=2 N_{A} \rho T V_{\mathrm{det}} \frac{d \Phi_{\nu}}{d E_{\nu}} e^{-E_{\nu} / E_{\nu}^{\mathrm{cut}}} \sigma_{\mathrm{CC}}\left(E_{\nu}\right) e^{-\tau_{\oplus}},
$$

then effectively traces the original neutrino flux, as the neutral-current (NC) events from all three flavors, which only contain $E_{\mathrm{sh}}=\left\langle y_{\mathrm{NC}}\right\rangle E_{\nu}$ (in addition to the smaller $\sigma_{\mathrm{NC}}$ ), only account for $<10 \%$ of the total signal.

Combined shower and muon observations would determine the $\nu_{\mu} /\left(\nu_{e}+\nu_{\tau}\right)$ ratio arriving from a source. This measured flavor ratio has many applications, as detailed in Refs. [10,11,51,52]. The atmospheric $\nu_{e}$ flux is only about $\sim 1 / 20$ of the $\nu_{\mu}$ background, which itself only adds to the shower background through the weaker NC channel, since $\nu_{\mu} \mathrm{CC}$ interactions are identifiable by the resulting muon tracks. We will not consider the prompt background, which is the only source of atmospheric $\nu_{\tau}$, as it only becomes important at very high energies [53]. These facts help offset the lower angular resolution of these events ( $\lesssim 10^{\circ}$ is expected for a Mediterranean detector [54], $\$ 25^{\circ}$ for IceCube [40]), which sets the background event rate. The improved shower resolution of a Mediterranean detector, as compared to IceCube (which is naturally limited by light scattering in ice), increases the value of showers in determining the high energy properties of a source.

\section{Neutrino spectroscopy}

To demonstrate the ability of a neutrino telescope to differentiate between various spectral properties, we first consider a general object with a fixed $\phi_{\gamma}=20 \times$ $10^{-12} \mathrm{TeV}^{-1} \mathrm{~cm}^{-2} \mathrm{~s}^{-1}$ (at $1 \mathrm{TeV}$ ) and assume a source declination of $\delta=+10^{\circ}$. The resulting up-going muon

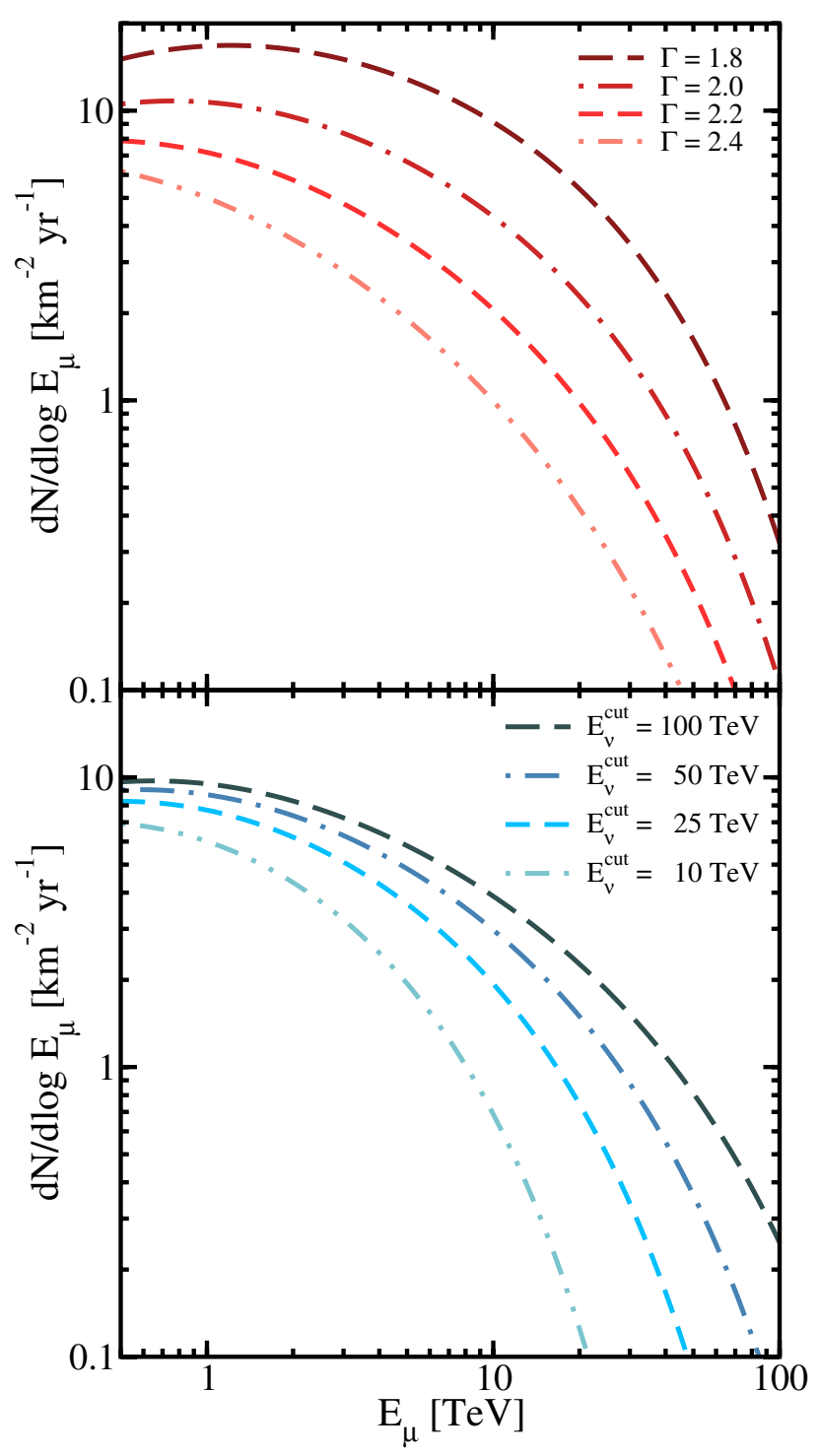

FIG. 2 (color online). A demonstration of the sensitivity of neutrino telescopes to varying spectral characteristics. Shown is the (base-10 log) differential $\left(\nu_{\mu}+\bar{\nu}_{\mu}\right)$-induced muon spectra for a pionic source spectrum with $\phi_{\gamma}=20 \times$ $10^{-12} \mathrm{TeV}^{-1} \mathrm{~cm}^{-2} \mathrm{~s}^{-1}$. In the upper panel, the lines of decreasing height correspond to spectral indices of $\Gamma=1.8,2.0,2.2$, and 2.4, with an exponential cutoff $E_{\nu}^{\text {cut }}=50 \mathrm{TeV}$. In the lower panel, the lines of decreasing height correspond to neutrino spectrum cutoffs of $100,50,25$, and $10 \mathrm{TeV}$, with a fixed spectral index of $\Gamma=2.1$. Each line corresponds to $1 \mathrm{yr}$ of observation. 
fluxes for a range of spectral indices and high energy exponential cutoffs, for $1 \mathrm{yr}$ of IceCube observation, are shown in Fig. 2. In the top panel, we consider spectral indices with $\Gamma=1.8,2.0,2.2$, and 2.4 , with $E_{\nu}^{\text {cut }}=$ $50 \mathrm{TeV}$. Note that the differences are not entirely due to differing integrated neutrino fluxes, as the rising $\sigma_{\nu N}$ results in higher event rates for harder spectra. In Ref. [8], similar results were obtained by normalizing to the integrated flux above $1 \mathrm{TeV}$. The bottom panel illustrates the effect of $100,50,25$, and $10 \mathrm{TeV}$ neutrino spectrum exponential cutoffs for a fixed index $(\Gamma=2.1)$. IceCube is well suited to isolate a similar point source located in the northern sky through $\nu_{\mu}$-induced muons within a short period of operation. Such a source would also be observable through shower events by a $\mathrm{km}^{3}$ Mediterranean detector. The improved shower detection capabilities of a Mediterranean detector would allow for a more direct measurement of the arriving neutrino spectrum, adding greatly to the science that can be extracted from $\mathrm{TeV}$ sources.

All of our calculations are done on an empirical basis using the average measured parameters of gamma-ray source spectra. All high energy exponential cutoffs that we will consider are given in terms of $E_{\nu}^{\text {cut }}\left(=1 / 2 E_{\gamma}^{\text {cut }}\right)$, to allow for a range of calculations to be compared with future experimental data. Angle-averaged FLUKA computations of atmospheric neutrinos past $E_{\nu}=1 \mathrm{TeV}$ are used to determine backgrounds [55]. For ease of comparing the expected signal to background and assessing total rates, we will mainly provide integrated rates above a given measured energy. Because the atmospheric spectrum is very steeply falling at these energies $\left(\sim E_{\nu}^{-3.5}\right)$, it is often advantageous to use a low energy event cutoff $\gtrsim 1 \mathrm{TeV}$ when calculating significance. Our method can also yield the measured muon and shower spectra directly. Spectral information should be used for maximum likelihood analysis of observed neutrino events in a neutrino telescope, to make the best use of measured energies in determining detection significance. This spectral information requires consideration of the energy resolution of the detector. In IceCube, the energy resolution for muons and showers is expected to be $\sim 20 \%$ and $\sim 10 \%$ in the logarithm of the energy, respectively [40,56], which does not significantly affect our conclusions.

Note that we present our results for an assumed muon effective area of $1 \mathrm{~km}^{2}$, and assumed muon angular resolution of $\leqslant 1^{\circ}$, both independent of energy. These results are close to the results of detector simulations, especially $\gtrsim 1 \mathrm{TeV}$, the primary area of interest [40,57]. Below $1 \mathrm{TeV}$, these assumptions are certainly overly optimistic; however, in that range, the atmospheric background is dominant, and so accuracy is of less importance. In regards to the angular resolution, our assumptions are somewhat too conservative (in particular, the improved resolution at higher energy will help reject the background). Part of our intent is to show the likely signals, and to encourage the experimentalists to optimize their detector designs in order to achieve the necessary sensitivity to observe Galactic $\mathrm{TeV}$ sources. These calculations are not meant to replace a more detailed study of neutrino source detectability, which would include time-dependent source locations, zenith-angle-dependent atmospheric backgrounds, angledependent detector sensitivities, event reconstruction methodologies, stochastic muon energy losses, source modeling, etc. Each prospective source should be subjected to such a full Monte Carlo simulation by the IceCube and Mediterranean collaborations.

\section{THE VELA COMPLEX}

Of the many known $\mathrm{TeV}$ gamma-ray sources, the shelltype SNR Vela Jr. (RX J0852.0-4622) is one of the most interesting. This southern-sky source has been observed at gamma-ray energies exceeding $10 \mathrm{TeV}$ by HESS. Their analysis is suggestive of a hadronic origin for the gamma-ray spectrum [26]. This source is very bright in gamma rays $(d \Phi / d E=21 \times$ $\left.10^{-12}(E / \mathrm{TeV})^{-2.1} \mathrm{TeV}^{-1} \mathrm{~cm}^{-2} \mathrm{~s}^{-1}\right)$, with well-defined regions of gamma-ray emission. Shell-type SNRs are considered to be the most likely sites of Galactic cosmic-ray proton acceleration [58]. As these source proton spectra are expected to be cut off near the knee $\left(\sim 3 \times 10^{15} \mathrm{eV}\right)$, a cutoff should also be present for gamma rays and neutrinos at a lower energy scale [38]. Thus, we calculate the expected neutrino-induced muon rate assuming a pionic spectrum with several neutrino spectrum exponential cutoffs $(50,25$, and $10 \mathrm{TeV})$, as shown in Fig. 3. When considering emission from the entire source extension, we must accept atmospheric background from a $\sim 7 \mathrm{deg}^{2}$ area. Comparing the source and background muon rates, this SNR is expected to be significantly detectable with a $\mathrm{km}^{3}$ Mediterranean detector. For the higher cutoffs, discovery may be possible in only a few years. These event rates also suggest that ANTARES may be able to find evidence of neutrino emission from this source [59].

A number of features make Vela Jr. unique among prospective $\mathrm{TeV}$ neutrino sources. The $\mathrm{TeV}$ emission is observed to originate from several regions which are separated by $\sim 2^{\circ}$. In addition to detecting the presence of neutrinos, the intensity of the measured muon spectrum may provide adequate statistics to allow an accurate location of neutrino-production sites. Thus, this unique gamma-ray morphology allows for the possible construction of a neutrino map of Vela Jr. This capability would enable a comparison of the production mechanism and high energy activity in different regions of the same source. When observing features of the neutrino source, the total atmospheric neutrino background enclosed is lower, which increases their individual detectability. An example of such a map can be seen in Ref. [60]. Further HESS observations should provide improved resolution of the $\mathrm{TeV}$ emission. 


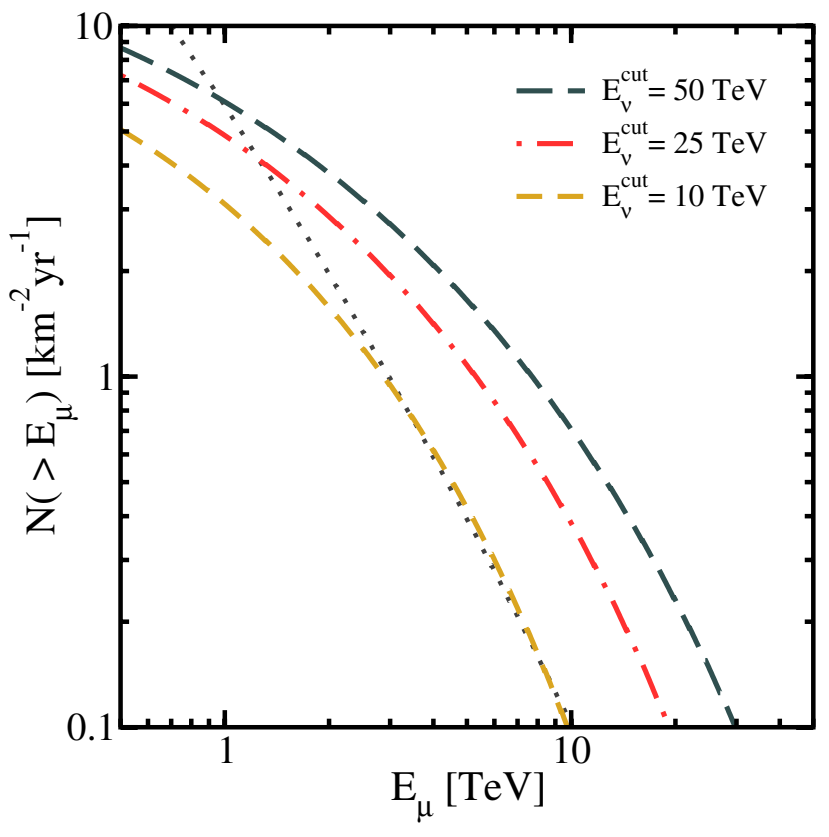

FIG. 3 (color online). Vela Jr. (RX J0852.0-4622)-Muons: Integrated $\left(\nu_{\mu}+\bar{\nu}_{\mu}\right)$-induced muon rates above a given measured muon energy. The long dashed, dot-dashed, and short dashed lines correspond to neutrino spectrum exponential cutoffs of 50,25 , and $10 \mathrm{TeV}$, respectively. The dotted line shows the expected atmospheric background in a $7 \operatorname{deg}^{2}$ bin, as discussed in the text. Rates are for $1 \mathrm{yr}$ of operation in a $\mathrm{km}^{3}$ Mediterranean detector.

Additionally, the brightness of the source may also make it possible to observe appreciable numbers of showers. As a consequence of the detected showers directly tracing the neutrino flux, spectral information may be found (even with a slightly higher background). Figure 4 shows the rate of showers for $1 \mathrm{yr}$ of observation, compared to the irreducible atmospheric background. With enough observation time, it may be possible to determine the neutrino flavor ratio from this source by observing the $\nu_{\mu}$ spectrum with a $\mathrm{km}^{3}$ Mediterranean detector and by also utilizing IceCube to effectively increase the shower volume (since shower events must be located within the detector) in measuring the $\nu_{e}+\nu_{\tau}$ flux.

Vela Jr. is coincident with a region of the larger Vela SNR. HESS has also reported the discovery of TeV gamma rays originating from the Vela PWN, referred to as Vela $X$ (of no relation to the HMXB Vela X-1) [27]. Vela X has a very hard spectrum, which is best fit with a spectral index of $\Gamma=1.45$ with an exponential cutoff beginning at $E_{\gamma}^{\text {cut }}=$ 13.8 TeV. In their discovery paper, HESS concludes that this emission is produced by an inverse Compton mechanism. An independent analysis has considered the possibility that this spectrum is produced hadronically, with an accompanying flux of neutrinos [28]. This source is sufficiently separated from Vela Jr. to independently test for neutrino emission through muons. If we assume the entire

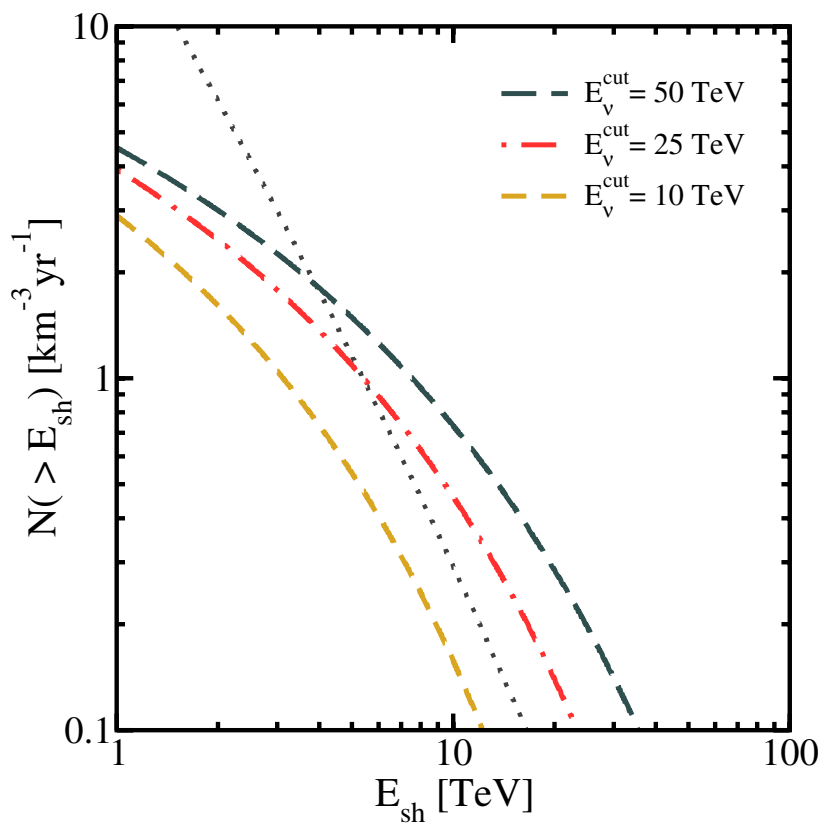

FIG. 4 (color online). Vela Jr. (J0852.0-4622)-Showers: Integrated neutrino-induced shower rates above a given measured shower energy, with signal lines as in Fig. 3. Note that a different energy scale is used for showers, as measurable shower events are assumed to have a $1 \mathrm{TeV}$ energy threshold. Background rates are for a circle of $10^{\circ}$ radius, corresponding to the shower angular resolution in a $\mathrm{km}^{3}$ Mediterranean detector. A significant reduction in background results from improved angular resolution. Rates are for $1 \mathrm{yr}$ of operation.

measured flux is pionic, then the expected $\nu_{\mu}$-induced muon rate is $N\left(E_{\mu}>1 \mathrm{TeV}\right) \sim 4.5 \mathrm{yr}^{-1}$, in a $\mathrm{km}^{3}$ Mediterranean detector, which would be relatively easy to measure. While our calculation may somewhat overstate the muon rate for such a hard spectrum, nevertheless, the nondetection of a significant neutrino event excess would indicate leptonic production.

Considering the large apparent size of the Vela SNR shell $\left(\sim 8^{\circ}\right)$, any $\mathrm{TeV}$ gamma-ray emission originating from it may be too diffuse to detect directly. Searching for it in neutrinos is a possibility. A northern-hemisphere detector would isolate any neutrino point source spectra located in the region through $\nu_{\mu}$-induced muons. Showers have the ability to observe this entire region simultaneously, raising the intriguing prospect of utilizing IceCube and $\mathrm{a} \mathrm{km}^{3}$ Mediterranean detector in concert to discover and study bright, but highly extended $\mathrm{TeV}$ neutrino sources.

\section{GALACTIC CENTER REGION}

The HESS discovery [29] of the region of Galactic Center diffuse emission (GCD) is important for neutrino, gamma-ray, and cosmic-ray astrophysics $[61,62]$. The spectrum of the GCD was measured over a very large region spanning the Galactic coordinates 
$|l|<0.8^{\circ}, \quad|b|<0.3^{\circ} \quad$ with $\quad d \Phi / d E=1.73 \times$ $10^{-8}(E / \mathrm{TeV})^{-2.29} \mathrm{TeV}^{-1} \mathrm{~cm}^{-2} \mathrm{~s}^{-1} \mathrm{sr}^{-1}$. A number of large, dense $\left(n_{H} \sim 10^{4} \mathrm{~cm}^{-3}\right)$ molecular clouds are known to fill this region [63]. Considering the vast extent of this hard emission ( $\sim$ few hundred pc across), along with the high target density and magnetic fields in the region, the only reasonable production mechanism for these gamma rays is neutral pion decay. The close morphological correlation between the $\mathrm{TeV}$ emission and the gas distribution is particularly compelling [29]. This TeV gamma-ray spectrum implies a local cosmic-ray spectrum that is much harder and 3-9 times denser than that of the CR flux as measured at Earth. The likely source of these cosmic-ray protons is Sgr A, which hosts the remnant of a recent supernova [64,65]. These pioneering conclusions were reached in Ref. [29].

In order to calculate the expected $\nu_{\mu}$-induced muon rate, we must first take into account the extent of the GCD source. The muon angular resolution of a neutrino telescope at these energies $\left(\sim 1^{\circ}\right)$ covers the entirety of the GCD. In effect, this region can be treated as a neutrino point source. Integrating over the $\sim 1 \mathrm{deg}^{2}$ source region, the total photon flux is $d \Phi / d E=5.2 \times$ $10^{-12}(E / \mathrm{TeV})^{-2.29} \mathrm{TeV}^{-1} \mathrm{~cm}^{-2} \mathrm{~s}^{-1}$. We define the differential flux at $1 \mathrm{TeV}$ as $\phi_{\mathrm{GCD}}=5.2 \times$ $10^{-12} \mathrm{TeV}^{-1} \mathrm{~cm}^{-2} \mathrm{~s}^{-1}$. We will not consider showers

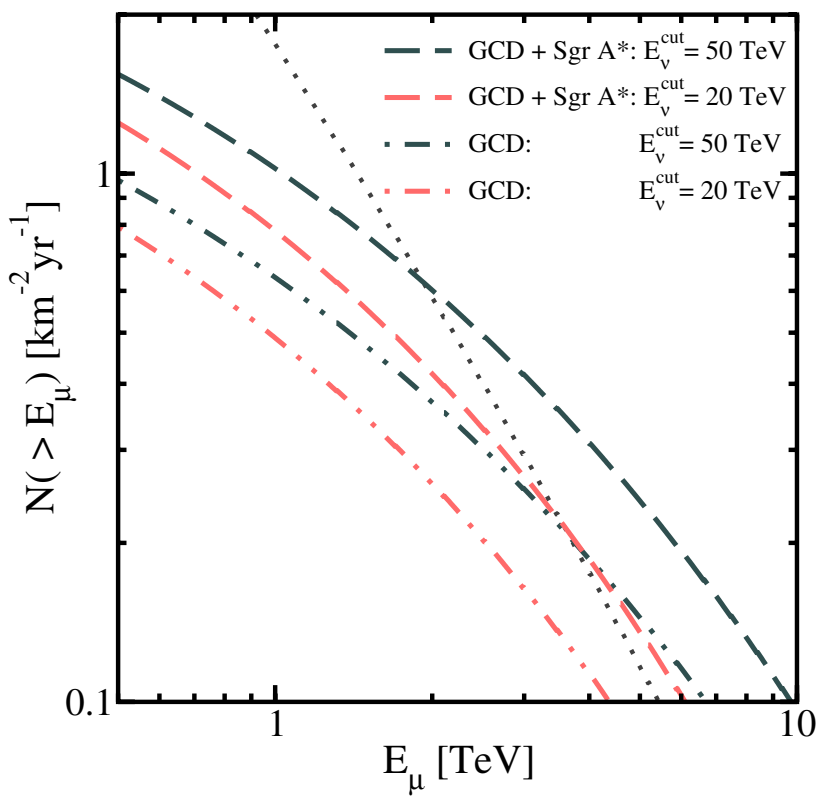

FIG. 5 (color online). Galactic Center region: Integrated $\left(\nu_{\mu}+\bar{\nu}_{\mu}\right)$-induced muon rates above a given measured muon energy. Shown are the rates from the Galactic Center diffuse source alone (dot-dashed line) and the result of including the Sgr $\mathrm{A}^{*}$ source (dashed line). Two cases are considered: a neutrino spectrum exponential cutoff $E_{\nu}^{\text {cut }}=50 \mathrm{TeV}$ and a more conservative cutoff of $20 \mathrm{TeV}$. The dotted line shows the estimated atmospheric background in a $3 \mathrm{deg}^{2}$ bin. Rates are for $1 \mathrm{yr}$ of observation in $\mathrm{a}^{3}$ Mediterranean detector. from the GCD; however, any such study should also take into account a similar diffuse emission around Sgr B [29].

We first consider the conservative assumption that the GCD spectrum exhibits an exponential neutrino spectrum cutoff $E_{\nu}^{\text {cut }}=20 \mathrm{TeV}$. As a cosmic-ray production site, we also consider a higher cutoff of $50 \mathrm{TeV}$. The expected muon rates are shown in Fig. 5. Even though its angle-integrated flux is larger, the GCD was revealed only after subtracting the previously discovered $\mathrm{TeV}$ emission from $\mathrm{Sgr} \mathrm{A}^{*}$ and a distant SNR [30,66]. The TeV gamma-ray flux from Sgr $\mathrm{A}^{*}$, which has $\phi_{\gamma} \sim 0.5 \phi_{\mathrm{GCD}}$, can reasonably be assumed to also be pionic, as the spectral index $(\Gamma=2.2)$ is close to the GCD value $[30,67]$. As the two sources are coincident, we combine their expected muon rates, as also shown in Fig. 5. Such an approach to calculating the total neutrino flux was also taken in Ref. [68], where the integrated muon rate was found. The declination of the source, along with the latitude of the detector, determine the fraction of the time that the source is below the horizon [24]. For the Galactic Center this is $\sim 0.7$, which is taken into account in Fig. 5. The signal rates shown in Fig. 5, when compared to the expected number of atmospheric events, suggest that this source is near the threshold of discovery for the lifetime of a $\mathrm{km}^{3}$ Mediterranean detector. Discovery may be hastened by utilizing maximum likelihood methods in comparing the expected signal with background for each muon event with a measured energy, as higher energy events have a much greater significance. Such an analysis is only possible with spectral information.

\section{A MULTITUDE OF TEV SOURCES}

Numerous discoveries of new gamma-ray sources should be expected from the HESS, MAGIC, and VERITAS air Cerenkov telescopes and the GLAST satellite [69-71]. However, the characteristics of the source spectra above $\sim 10 \mathrm{TeV}$ are difficult to observe. RX J1713-3946 is also a shell-type SNR, first observed in the $\mathrm{TeV}$ regime by CANGAROO [72]. It has since been observed twice by HESS with greatly improved statistics. We consider both HESS observations to illustrate the potential of neutrino telescopes to determine the high energy behavior of $\mathrm{TeV}$ sources. The flux was initially reported up to $10 \mathrm{TeV}$ as a power law $(\Gamma \sim 2.19)$, with analysis favoring hadronic production [31]. Subsequent observations revealed a spectrum that extends to at least $40 \mathrm{TeV}$ with steepening past $\sim 10 \mathrm{TeV}$ that can be fit by either an energy dependent spectral index or an exponential cutoff [32]. Previous neutrino studies of this source were inspired first by the CANGAROO detection [17] and later by utilizing the more accurate 2004 HESS spectrum along with a more detailed calculation [18]. We analyze the $\nu_{\mu}$-induced muon spectra from these two data sets independently, assuming $E_{\nu}^{\text {cut }}=50 \mathrm{TeV}$ for the 2004 data. As seen in Table I, the integrated rates above $1 \mathrm{TeV}$ are similar. However, at higher energies calculations based upon the 
TABLE I. HESS Sources With Probable Counterparts: Integrated $\left(\nu_{\mu}+\bar{\nu}_{\mu}\right)$-induced muon rates, assuming a pionic spectrum, for a $1 \mathrm{TeV} E_{\mu}$ threshold and neutrino spectrum exponential cutoff $E_{\nu}^{\text {cut }}$, for $1 \mathrm{yr}$ of $\mathrm{km}^{3}$ Mediterranean detector operation (unless noted), accounting for observable time below the horizon. The HESS differential flux at $1 \mathrm{TeV}$ is given in terms of $10^{-12} \mathrm{TeV}^{-1} \mathrm{~cm}^{-2} \mathrm{~s}^{-1}$, with spectral index $\Gamma$.

\begin{tabular}{|c|c|c|c|c|}
\hline Source & $\phi_{\gamma}$ & $\Gamma$ & $E_{\nu}^{\text {cut }}(\mathrm{TeV})$ & $N_{\mu}(>1 \mathrm{TeV})$ \\
\hline Vela Jr. & 21.0 & 2.1 & 10 & 3.1 \\
\hline \multirow[t]{2}{*}{ (RX J0852.0-4622) } & & & 25 & 4.9 \\
\hline & & & 50 & 6.1 \\
\hline \multirow[t]{2}{*}{ GC Diffuse } & 5.2 & 2.29 & 20 & 0.5 \\
\hline & & & 50 & 0.7 \\
\hline \multirow[t]{2}{*}{ (+ GC Source) } & & & 20 & 0.8 \\
\hline & & & 50 & 1.0 \\
\hline \multirow[t]{2}{*}{ RX J1713.7-3946 } & 15.0 & 2.19 & 50 & 2.8 \\
\hline & 20.4 & 1.98 & 6 & 2.2 \\
\hline Vela X & 9.0 & 1.45 & 7 & 4.5 \\
\hline Crab (IceCube) & 33.0 & 2.57 & 50 & 2.7 \\
\hline \multirow[t]{2}{*}{ HESS J1514-591 } & 5.7 & 2.27 & 25 & 0.9 \\
\hline & & & 50 & 1.1 \\
\hline \multirow[t]{2}{*}{ HESS J1616-508 } & 6.0 & 2.35 & 10 & 0.5 \\
\hline & & & 50 & 0.9 \\
\hline \multirow[t]{2}{*}{ HESS J1632-478 } & 5.5 & 2.12 & 10 & 0.8 \\
\hline & & & 50 & 1.5 \\
\hline \multirow[t]{2}{*}{ HESS J1634-472 } & 2.0 & 2.38 & 10 & 0.2 \\
\hline & & & 50 & 0.3 \\
\hline \multirow[t]{2}{*}{ HESS J1640-465 } & 3.0 & 2.42 & 10 & 0.2 \\
\hline & & & 50 & 0.4 \\
\hline \multirow[t]{2}{*}{ HESS J1745-303 } & 2.5 & 1.8 & 10 & 0.5 \\
\hline & & & 50 & 1.2 \\
\hline \multirow[t]{2}{*}{ HESS J1804-216 } & 4.7 & 2.72 & 10 & 0.1 \\
\hline & & & 50 & 0.2 \\
\hline \multirow[t]{2}{*}{ HESS J1813-178 } & 2.7 & 2.09 & 10 & 0.3 \\
\hline & & & 50 & 0.5 \\
\hline \multirow[t]{2}{*}{ HESS J1825-137 } & 6.0 & 2.46 & 25 & 0.4 \\
\hline & & & 50 & 0.5 \\
\hline \multirow[t]{2}{*}{ HESS J1834-087 } & 2.5 & 2.45 & 10 & 0.1 \\
\hline & & & 50 & 0.2 \\
\hline \multirow[t]{2}{*}{ HESS J1837-069 } & 5.0 & 2.27 & 10 & 0.3 \\
\hline & & & 50 & 0.6 \\
\hline
\end{tabular}

old power law spectrum exhibit a deviation from the new data. From the 2004 data, we find $N\left(E_{\mu}>10 \mathrm{TeV}\right) \sim$ $0.3 \mathrm{yr}^{-1}$, while the 2005 data yields a rate only $\sim 1 / 10$ of this, which highlights the sensitivity of neutrino observations to the spectrum at the highest energies. Based on either spectrum, this SNR should be detectable in a $\mathrm{km}^{3}$ Mediterranean detector.

Amongst the HESS catalog of $\mathrm{TeV}$ sources are several with no observed counterpart at any other wavelength. Explaining the origin of these sources is a theoretical challenge. It is possible that they represent a nontrivial new class of astrophysical objects. With so little being known about them at the present, the observation of neutrinos from these sources would yield invaluable insight
TABLE II. Unidentified HESS Sources: Integrated $\left(\nu_{\mu}+\bar{\nu}_{\mu}\right)$ induced muon rates, assuming a pionic spectrum, for a $1 \mathrm{TeV} E_{\mu}$ threshold and neutrino spectrum exponential cutoff $E_{\nu}^{\text {cut }}$, for $1 \mathrm{yr}$ of $\mathrm{km}^{3}$ Mediterranean detector operation, accounting for observable time below the horizon. The HESS differential flux at $1 \mathrm{TeV}$ is given in terms of $10^{-12} \mathrm{TeV}^{-1} \mathrm{~cm}^{-2} \mathrm{~s}^{-1}$, with spectral index $\Gamma$.

\begin{tabular}{ccccc}
\hline \hline Source & $\phi_{\gamma}$ & $\Gamma$ & $E_{\nu}^{\text {cut }}(\mathrm{TeV})$ & $N_{\mu}(>1 \mathrm{TeV})$ \\
\hline HESS J1303-631 & 4.3 & 2.44 & 10 & 0.3 \\
& & & 50 & 0.5 \\
HESS J1614-518 & \multirow{2}{*}{2.0} & 2.46 & 10 & 0.5 \\
& & & 50 & 0.8 \\
HESS J1702-420 & \multirow{2}{*}{2.5} & \multirow{2}{*}{2.31} & 25 & 0.3 \\
& & & 50 & 0.4 \\
HESS J1708-410 & \multirow{2}{*}{1.5} & \multirow{2}{*}{2.34} & 10 & 0.1 \\
& & & 50 & 0.2 \\
\hline \hline
\end{tabular}

into their nature. The first of these sources is HESS J1303-631 [34]. Chandra observations revealed no likely $\mathrm{x}$-ray counterpart [73]. This source is always visible to a Mediterranean detector. Three sources were also found in the HESS survey of the Galactic plane that remain unidentified: HESS J1614-518, HESS J1702-420, and HESS J1708-410 [33]. The spectral information and resulting integrated $\nu_{\mu}$-induced muon rates from these sources are given in Table II. It has been proposed that HESS J1303631 is a remnant of a Galactic gamma-ray burst (GRB), with a harder $(\Gamma \sim 2.2)$ and more extended emission than has been observed [74]. From the given predictions of the proposed hadronic source model, we would expect $N\left(E_{\mu}>1 \mathrm{TeV}\right) \sim 2.1 \mathrm{yr}^{-1}$, which is potentially verifiable with a $\mathrm{km}^{3}$ Mediterranean detector. From the measured spectra, detecting these sources individually may be difficult; however, the stacking method may prove advantageous. Stacking effectively increases the total observation rate by combining the individual event rates from identical sources [75-78]. Stacking of the observed muon data may improve the prospects of determining whether neutrinos are present and help unveil the mystery of these unknown sources.

Other Galactic $\mathrm{TeV}$ sources with probable counterparts may also be examined as potential neutrino sources. Many new sources were found as a result of the HESS inner Galactic plane survey, some of which may be SNRs [33]. MAGIC and VERITAS observations will reveal sources in the outer regions of the plane, which has yet to be examined with an instrument of their sensitivity. Assuming that the gamma-ray spectra already observed by HESS are pionic, the calculated neutrino event rates for a number of sources are shown in Table I. Several of these have sufficient rates to consider as possible neutrino sources independently. However, it would be difficult to significantly detect dimmer sources over the atmospheric background. Stacking, in combination with a maximum 
likelihood spectral analysis, may be used to examine the entire group, or subgroups, of the lower-flux sources.

\section{A TEV NEUTRINO BEACON}

It would be beneficial to have a bright source of $\mathrm{TeV}$ neutrinos that can be seen by both IceCube and a Mediterranean detector. The ideal source for this purpose would have a declination in the range $+10^{\circ} \lesssim \delta \lesssim+30^{\circ}$, which also has the benefit of reduced neutrino attenuation in Earth at high energies, resulting in increased muon and shower event rates. The Crab nebula is already an accepted standard candle in $\mathrm{TeV}$ gamma-ray astronomy. From HESS measurements [79], if the entire TeV spectrum could be attributed to hadronic processes, our methods yield an event rate of $N\left(E_{\mu}>1 \mathrm{TeV}\right) \sim 2.7 \mathrm{yr}^{-1}$ in IceCube. The Crab spectrum is relatively soft and well described by leptonic processes [80], although AMANDA observations hint at a neutrino signal [81]. A pionic component may be uncovered by a significant observation of neutrino events.

The Vela complex that we have already discussed is located in the southern sky. Only after extensive observations was the existence of Vela Jr. confirmed [82]. It would not be surprising, then, for a similarly bright $\mathrm{TeV}$ source to be discovered in the northern sky (perhaps directly through neutrino events). The spectrum for such an object was calculated near the end of Sec. III for a variety of spectral indices and cutoffs. Future gamma-ray observations of this declination range in the $\mathrm{TeV}$ regime may reveal another important source for neutrino astronomy.

Multiwavelength observations of such a source would add a rung to a TeV neutrino "distance ladder". Cosmicray interactions in the Earth's atmosphere generate the nearest guaranteed neutrino source [83,84]. Similar processes in the atmosphere of the Sun, which is at a wellknown distance, are quite likely to produce $\mathrm{TeV}$ neutrinos as well [85-87]. Of the prospective sources, the Vela SNR is estimated to be only $\sim 250-300$ pc from Earth, while Vela Jr. may be even closer [88-90]. RX J1713-3946 has a distance estimate of $\sim 1 \mathrm{kpc}$ [91]. At $\sim 8 \mathrm{kpc}$, the GCD is the most distant guaranteed source with a confirmed distance. AGN, if they produce measurable fluxes of neutrinos, would provide a range of very remote point sources. Measurements of neutrinos from a variety of distances (ideally with flavor ratios) would provide important information for testing neutrino properties at increasing $L / E$ (distance/energy) [22,92-100].

\section{EGRET, THE TEV EXCESS, AND THE GREAT UNKNOWN}

The third EGRET catalog contains many unidentified gamma-ray sources detected in the $\mathrm{MeV}-\mathrm{GeV}$ range [101]. In the Whipple survey of a selected group of such EGRET sources, upper limits on high energy gamma-ray emissions from a number of sources were determined [102]. Two of
TABLE III. Two Unidentified EGRET Sources: Integrated $\left(\nu_{\mu}+\bar{\nu}_{\mu}\right)$-induced muon rates, assuming a pionic spectrum, for a given $E_{\mu}$ threshold and neutrino spectrum exponential cutoff $E_{\nu}^{\text {cut }}$ (in TeV), for $1 \mathrm{yr}$ of IceCube operation. The assumed differential photon flux at $1 \mathrm{TeV}$ is given in terms of $10^{-12} \mathrm{TeV}^{-1} \mathrm{~cm}^{-2} \mathrm{~s}^{-1}$, with spectral index $\Gamma$.

\begin{tabular}{cccccc}
\hline \hline Source & $\phi_{\gamma}$ & $\Gamma$ & $E_{\nu}^{\text {cut }}$ & $N_{\mu}(>0.5 \mathrm{TeV})$ & $N_{\mu}(>1 \mathrm{TeV})$ \\
\hline 3EG J1337+5029 & 20 & 2.2 & 10 & 4.1 & 2.5 \\
& & & 50 & 6.9 & 4.7 \\
3EG J2227+6122 & \multirow{2}{*}{10} & 2.0 & 10 & 3.0 & 1.9 \\
& & & 50 & 5.5 & 4.0 \\
\hline \hline
\end{tabular}

these sources, 3EG J1337+5029 and 3EG J2227+6122, possessed measured excesses that were suggestive of gamma-ray emission [103]. The upper limits placed on these sources do not significantly constrain the EGRET data. We consider the possibility that these spectra are pionic and continue into the $\mathrm{TeV}$ regime. Spectral information, cutoffs, and muon rates are given in Table III. These would be readily detectable by IceCube. We note that 3EG J2227+6122 is roughly coincident with a position in the three-year AMANDA point source survey which exhibits a modest excess of neutrino events [12]. A less significant excess still appears in newer data [104]. In general, a maximum likelihood analysis assuming the spectrum and normalization of such bright EGRET sources extended into the $\mathrm{TeV}$ range may prove useful for neutrino searches. Future observations by GLAST, VERITAS, and MAGIC will provide valuable additional spectral information.

Recently, Milagro announced the detection of diffuse $\mathrm{TeV}$ emission from a $\sim 600 \mathrm{deg}^{2}$ patch of the Galactic plane that contains ten unidentified EGRET point sources [105]. This measurement places high energy constraints on the spectra of these ten sources, as discussed in Ref. [36]. Consequently, these spectra, many of which are quite hard, cannot all simply extrapolate to $\mathrm{TeV}$ energies. HEGRA discovered an unidentified $\mathrm{TeV}$ source in this region, however, it can account for only a small fraction of the Milagro flux [106]. Located in the northern sky, this region is accessible to observations by IceCube. If this emission is pionic and truly diffuse, then it would be very difficult to detect through neutrino-induced muons [107]. Assuming that the entire flux is instead divided among several point sources, the expected muon rate can be calculated. A detailed Milagro study of the Cygnus region revealed a significant $\mathrm{TeV}$ flux [108]. Assigning all of the Milagro 3.5 TeV flux to a single source and extrapolating with a typical $\Gamma=2.2$ gamma-ray spectrum (out to $100 \mathrm{TeV}$ ), we arrive at a limiting case for the possible neutrino emission from the region. For a pionic spectrum, the calculated $\nu_{\mu}$-induced muon rate is $N\left(E_{\mu}>1 \mathrm{TeV}\right) \sim 2.3 \mathrm{yr}^{-1}$ in IceCube. Future measurements of this region by Milagro, GLAST, VERITAS, and MAGIC will determine whether multiple sources contribute to this diffuse flux. 


\section{DISCUSSION AND CONCLUSIONS}

Upcoming neutrino telescopes will deliver the first direct evidence concerning the production mechanism of $\mathrm{TeV}$ gamma-ray sources. Following the pioneering AMANDA [109] and Baikal [110] efforts, the next generation IceCube [6] and Mediterranean [7] $\mathrm{km}^{3}$ detectors will reach the scale necessary to examine the Galactic sources discussed here. Hadronic mechanisms would be confirmed through neutrino detection, while a significant absence of neutrino events would imply leptonic processes. They will be able to probe the expected high energy spectral cutoffs that would otherwise be unobservable. It has been suggested that the cutoff in a SNR may evolve with age [111]. Finding these cutoffs with neutrinos may also yield valuable new information in this regard.

Combined, IceCube and $\mathrm{a} \mathrm{km}^{3}$ Mediterranean detector will provide continuous, all-sky coverage and can be used together to detect neutrino-induced muons and showers from $\mathrm{TeV}$ sources. The good angular resolution for muon events can be used to precisely locate a source in neutrinos. Showers, and contained muon events, provide accurate reconstruction of the source spectrum at energies beyond the reach of gamma-ray telescopes. These measurements will provide important information concerning the origin of high energy Galactic cosmic rays, potentially directly observing the source population responsible for production up to the knee at $\sim 3 \times 10^{15} \mathrm{eV}$.

The shell-type SNR Vela Jr. (RX J0852.0-4622) is an intriguing prospective source of $\mathrm{TeV}$ neutrinos. The extent and intensity of the $\mathrm{TeV}$ emission make this, as well as the Vela complex as a whole, a unique target for neutrino telescopes. The confirmation of hadronic/leptonic processes through detection/nondetection of neutrino events from Vela $\mathrm{X}$ and the possible detection of neutrinos through shower events from the Vela SNR shell are exciting possibilities.

While it is expected that other sources may eventually be more compelling, only the Galactic Center diffuse emission can presently be claimed to possess a guaranteed neutrino flux. The detection of this neutrino flux will give further insight into the complex processes occurring in the Galactic Center region. When we consider that, to date, no high energy astrophysical neutrinos have ever been positively detected, the importance of such a guaranteed flux of $\mathrm{TeV}$ neutrinos is difficult to overstate.

Included in the catalog of $\mathrm{TeV}$ sources are several that remain unidentified. As more observations are undertaken by the HESS, MAGIC, and VERITAS telescopes, this number is expected to increase. Discovering neutrino fluxes from these sources would provide invaluable information concerning their nature. As new $\mathrm{TeV}$ gamma-ray sources are discovered, the pool of potential $\mathrm{TeV}$ neutrino sources increases. While some of these may not be significant alone, when grouped into classes, stacking may in- crease the potential for neutrino studies. An even larger number of unidentified sources remains in the $\mathrm{GeV}$ regime from EGRET observations. Many of these sources have intense, hard spectra. If any of these sources, some of which are visible to IceCube, have spectra that extrapolate into the $\mathrm{TeV}$ regime and are pionic, they would have abundant fluxes of neutrinos.

In summary, the prospects for the near-term first discoveries of Galactic $\mathrm{TeV}$ neutrino sources are very good. Importantly, this conclusion is empirically based on the measured spectra of bright Galactic $\mathrm{TeV}$ gamma-ray sources. For some of these, there are very compelling independent arguments that the observed gamma rays arise from neutral pion decays, meaning that they must be accompanied by neutrinos. It is essential to test this directly for these sources, as well as for others where the possibility of neutrino emission is uncertain. We emphasize the importance of using the measured muon energy spectra to discriminate against the quickly falling atmospheric neutrino backgrounds. For example, a single event near $10 \mathrm{TeV}$ from a source direction is almost certainly signal, while an event near $1 \mathrm{TeV}$ has a much higher probability of being background. This fact alone could be enough to help establish discovery. Because of the amplifying factors of neutrino cross section and muon range, neutrino detectors have better reach to the highest energies in the source spectra, as compared to gamma-ray telescopes, which can make precise measurements at lower energies. This complementarity can be exploited to help solve the long-standing puzzle of the origin of the Galactic cosmic rays.

For all of the sources discussed here, the rates in $\mathrm{km}^{3}$ neutrino telescopes are relatively small, at most $\sim 1-10$ events/year, though we have argued that even these small rates could have a powerful impact. While it remains possible that various uncertainties and detector limitations may make these observations even more challenging, they might also be better than described here. If the source spectra extrapolate to higher energies than we have assumed, or especially if the emitted $\mathrm{TeV}$ gamma-ray spectra have been diminished by absorption in the sources, which is quite possible, then the neutrino detection event rates could be significantly larger than shown here. Given the potentially unique power of neutrino astronomy, we can only hope that nature has been so kind.

\section{ACKNOWLEDGMENTS}

We are grateful to Felix Aharonian, Alexander Kappes, Paolo Lipari, Francesco Vissani, Casey Watson, and Hasan Yüksel for helpful comments and discussions. M. D. K. acknowledges support from The Ohio State University Department of Physics. J.F. B. was supported by the National Science Foundation under CAREER Grant No. PHY-0547102, and by The Ohio State University. 
[1] J. A. Hinton (HESS Collaboration), New Astron. Rev. 48, 331 (2004).

[2] F. W. Stecker, Astrophys. J. 228, 919 (1979).

[3] T. K. Gaisser, F. Halzen, and T. Stanev, Phys. Rep. 258, 173 (1995); 271, 355(E) (1996).

[4] J. G. Learned and K. Mannheim, Annu. Rev. Nucl. Part. Sci. 50, 679 (2000).

[5] F. Halzen and D. Hooper, Rep. Prog. Phys. 65, 1025 (2002).

[6] J. Ahrens et al. (IceCube Collaboration), Nucl. Phys. B, Proc. Suppl. 118, 388 (2003).

[7] U.F. Katz, astro-ph/0606068; http://www.km3net.org.

[8] P. Lipari, astro-ph/0605535.

[9] F. Aharonian, "Galactic sources of cosmic rays and neutrinos," talk at Neutrino 2006, http://neutrinosantafe06. com/; A. Kappes, J. Hinton, Ch. Stegmann, and F. Aharonian, astro-ph/0607286.

[10] J. F. Beacom, N. F. Bell, D. Hooper, S. Pakvasa, and T. J. Weiler, Phys. Rev. D 68, 093005 (2003); 72, 019901(E) (2005).

[11] G. Barenboim and C. Quigg, Phys. Rev. D 67, 073024 (2003).

[12] M. Ackermann et al. (AMANDA Collaboration), Phys. Rev. D 71, 077102 (2005).

[13] A. M. Hillas, J. Phys. G 31, R95 (2005)

[14] E. W. Kolb, M. S. Turner, and T. P. Walker, Phys. Rev. D 32, 1145 (1985); 33, 859(E) (1986).

[15] R. M. Crocker, F. Melia, and R. R. Volkas, Astrophys. J. 622, L37 (2005).

[16] J. Candia, J. Cosmol. Astropart. Phys. 11 (2005) 002.

[17] J. Alvarez-Muniz and F. Halzen, Astrophys. J. 576, L33 (2002).

[18] M. L. Costantini and F. Vissani, Astropart. Phys. 23, 477 (2005).

[19] W. Bednarek, G. F. Burgio, and T. Montaruli, New Astron. Rev. 49, 1 (2005).

[20] F. A. Aharonian, L. A. Anchordoqui, D. Khangulyan, and T. Montaruli, J. Phys.: Conf. Ser. 39, 408 (2006).

[21] L. A. Anchordoqui, H. Goldberg, F. Halzen, and T. J. Weiler, Phys. Lett. B 593, 42 (2004).

[22] L. A. Anchordoqui, H. Goldberg, M. C. Gonzalez-Garcia, F. Halzen, D. Hooper, S. Sarkar, and T. J. Weiler, Phys. Rev. D 72, 065019 (2005).

[23] G. Bertone, A. R. Zentner, and J. Silk, Phys. Rev. D 72, 103517 (2005).

[24] G. Bertone, Phys. Rev. D 73, 103519 (2006).

[25] I. F. M. Albuquerque, J. Lamoureux, and G. F. Smoot, Astrophys. J. Suppl. Ser. 141, 195 (2002).

[26] F. Aharonian et al. (HESS Collaboration), Astron. Astrophys. 437, L7 (2005)

[27] F. Aharonian et al. (HESS Collaboration), Astron. Astrophys. 448, L43 (2006).

[28] D. Horns, F. Aharonian, A. Santangelo, A. I. D. Hoffmann, and C. Masterson, Astron. Astrophys. 451, L51 (2006).

[29] F. Aharonian et al. (HESS Collaboration), Nature (London) 439, 695 (2006).

[30] F. Aharonian et al. (HESS Collaboration), Astron. Astrophys. 425, L13 (2004).

[31] F. A. Aharonian et al. (HESS Collaboration), Nature (London) 432, 75 (2004).

[32] F. Aharonian et al. (HESS Collaboration), Astron.
Astrophys. 449, 223 (2006).

[33] F. Aharonian et al. (HESS Collaboration), Astrophys. J. 636, 777 (2006).

[34] F. Aharonian et al. (HESS Collaboration), Astron. Astrophys. 439, 1013 (2005).

[35] N. Gehrels, D. J. Macomb, D. L. Bertsch, D. J. Thompson, and R. C. Hartman, Nature (London) 404, 363 (2000).

[36] T. Prodanovic, B.D. Fields, and J.F. Beacom, astro-ph/ 0603618.

[37] T. K. Gaisser, Cosmic Rays and Particle Physics (Cambridge University Press, Cambridge, England, 1990).

[38] S. R. Kelner, F. A. Aharonian, and V. V. Bugayov, astro-ph/ 0606058.

[39] L. A. Anchordoqui, H. Goldberg, F. Halzen, and T.J. Weiler, Phys. Lett. B 621, 18 (2005).

[40] J. Ahrens et al. (IceCube Collaboration), Astropart. Phys. 20, 507 (2004).

[41] R.P. Kokoulin and A. A. Petrukhin, Nucl. Instrum. Methods Phys. Res., Sect. A 263, 468 (1988).

[42] P. Lipari and T. Stanev, Phys. Rev. D 44, 3543 (1991).

[43] A.P. Chikkatur et al. (NuTeV/CCFR Collaboration), Z. Phys. C 74, 279 (1997).

[44] L. Anchordoqui and F. Halzen, hep-ph/0510389.

[45] R. Gandhi, C. Quigg, M. H. Reno, and I. Sarcevic, Phys. Rev. D 58, 093009 (1998).

[46] R. Gandhi, C. Quigg, M.H. Reno, and I. Sarcevic, Astropart. Phys. 5, 81 (1996).

[47] M. C. Gonzalez-Garcia, F. Halzen, and M. Maltoni, Phys. Rev. D 71, 093010 (2005).

[48] S. I. Dutta, M. H. Reno, I. Sarcevic, and D. Seckel, Phys. Rev. D 63, 094020 (2001).

[49] J. F. Beacom, P. Crotty, and E. W. Kolb, Phys. Rev. D 66, 021302 (2002).

[50] T. K. Gaisser and T. Stanev, Phys. Rev. D 31, 2770 (1985).

[51] P. D. Serpico and M. Kachelriess, Phys. Rev. Lett. 94, 211102 (2005).

[52] W. Winter, hep-ph/0604191.

[53] J. F. Beacom and J. Candia, J. Cosmol. Astropart. Phys. 11 (2004) 009.

[54] B. Hartmann, astro-ph/0606697.

[55] G. Battistoni, A. Ferrari, T. Montaruli, and P. R. Sala, hep$\mathrm{ph} / 0305208$.

[56] M. Kowalski, J. Cosmol. Astropart. Phys. 05 (2005) 010.

[57] M. Circella, 29th International Cosmic Ray Conference, Pune (Tata Institute of Fundamental Research, Mumbai, 2005), Vol. 5, p. 47.

[58] G. Rowell, F. Aharonian, and A. Plyasheshnikov, astro-ph/ 0512523.

[59] E. Aslanides et al. (ANTARES Collaboration), astro-ph/ 9907432.

[60] U. F. Katz, Prog. Part. Nucl. Phys. 57, 273 (2006).

[61] I. Busching, O.C. de Jager, and J. Snyman, astro-ph/ 0602193.

[62] S. M. Liu, F. Melia, V. Petrosian, and M. Fatuzzo, astro$\mathrm{ph} / 0603137$.

[63] M. Tsuboi, T. Handa, and N. Ukita, Astrophys. J. Suppl. Ser. 120, 1 (1999).

[64] Y. Maeda et al., Astrophys. J. 570, 671 (2002).

[65] C. L. Fryer, G. Rockefeller, A. Hungerford, and F. Melia, Astrophys. J. 638, 786 (2006). 
[66] F. Aharonian et al. (HESS Collaboration), Astron. Astrophys. 432, L25 (2005).

[67] J. Albert et al. (MAGIC Collaboration), Astrophys. J. 638, L101 (2006).

[68] V. Cavasinni, D. Grasso, and L. Maccione, astro-ph/ 0604004.

[69] C. Baixeras (MAGIC Collaboration), Nucl. Phys. B, Proc. Suppl. 114, 247 (2003).

[70] T. C. Weekes et al., Astropart. Phys. 17, 221 (2002).

[71] N. Gehrels and P. Michelson, Astropart. Phys. 11, 277 (1999).

[72] R. Enomoto et al., Nature (London) 416, 823 (2002).

[73] R. Mukherjee and J. P. Halpern, Astrophys. J. 629, 1017 (2005).

[74] A. Atoyan, J. Buckley, and H. Krawczynski, Astrophys. J. 642, L153 (2006)

[75] W. N. Brandt et al., Astrophys. J. 122, 2810 (2001).

[76] A. N. Cillis, D.F. Torres, and O. Reimer, Astrophys. J. 621, 139 (2005).

[77] K. Brand et al., Astrophys. J. 626, 723 (2005).

[78] A. Achterberg et al. (IceCube Collaboration), astro-ph/ 0509330.

[79] C. Masterson, W. Benbow, S. Funk, and S. Gillessen (HESS Collaboration), AIP Conf. Proc. No. 745 (AIP, New York, 2005), p. 617.

[80] F. Aharonian et al. (HEGRA Collaboration), Astrophys. J. 614, 897 (2004)

[81] M. Ackermann, E. Bernardini, and T. Hauschildt (IceCube Collaboration), 29th International Cosmic Ray Conference, Pune (Tata Institute of Fundamental Research, Mumbai, 2005), Vol. 5, p. 5.

[82] B. Aschenbach, Nature (London) 396, 141 (1998).

[83] V. A. Balkanov et al., Astropart. Phys. 12, 75 (1999).

[84] J. Ahrens et al. (AMANDA Collaboration), Phys. Rev. D 66, 012005 (2002).

[85] D. Seckel, T. Stanev, and T. K. Gaisser, Astrophys. J. 382, 652 (1991).

[86] I. V. Moskalenko, S. Karakula, and W. Tkaczyk, Astron. Astrophys. 248, L5 (1991).

[87] G. Ingelman and M. Thunman, Phys. Rev. D 54, 4385 (1996).

[88] A. N. Cha, K. R. Sembach, and A. C. Danks, Astrophys. J. 515, L25 (1999).
[89] R. Dodson, D. Legge, J.E. Reynolds, and P. M. McCulloch, Astrophys. J. 596, 1137 (2003).

[90] B. Aschenbach, A. F. Iyudin, and V. Schoenfelder, Astron. Astrophys. 350, 997 (1999).

[91] Y. Moriguchi, K. Tamura, Y. Tawara, H. Sasago, K. Yamaoka, T. Onishi, and Y. Fukui, Astrophys. J. 631, 947 (2005).

[92] J. F. Beacom, N. F. Bell, D. Hooper, S. Pakvasa, and T. J. Weiler, Phys. Rev. Lett. 90, 181301 (2003).

[93] J. F. Beacom, N. F. Bell, D. Hooper, S. Pakvasa, and T. J. Weiler, Phys. Rev. D 69, 017303 (2004).

[94] P. Keranen, J. Maalampi, M. Myyrylainen, and J. Riittinen, Phys. Lett. B 574, 162 (2003).

[95] J.F. Beacom, N.F. Bell, D. Hooper, J. G. Learned, S. Pakvasa, and T. J. Weiler, Phys. Rev. Lett. 92, 011101 (2004).

[96] D. Hooper, D. Morgan, and E. Winstanley, Phys. Rev. D 72, 065009 (2005).

[97] D. Hooper, D. Morgan, and E. Winstanley, Phys. Lett. B 609, 206 (2005).

[98] J. Christian, Phys. Rev. D 71, 024012 (2005).

[99] K. R.S. Balaji, G. Couture, and C. Hamzaoui, hep-ph/ 0605066

[100] N. Gupta and H. S. Mani, J. Phys. G 31, 599 (2005).

[101] R. C. Hartman et al. (EGRET Collaboration), Astrophys. J. Suppl. Ser. 123, 79 (1999).

[102] S. J. Fegan et al., Astrophys. J. 624, 638 (2005).

[103] S. J. Fegan and T. C. Weekes, Astrophys. Space Sci. 297, 431 (2005).

[104] P. O. Hulth (IceCube Collaboration), astro-ph/0604374.

[105] R. W. Atkins et al. (Milagro Collaboration), Phys. Rev. Lett. 95, 251103 (2005).

[106] F. Aharonian et al. (HEGRA Collaboration), astro-ph/ 0501667.

[107] J. L. Kelley (IceCube Collaboration), astro-ph/0509546.

[108] A. J. Smith (Milagro Collaboration), 29th International Cosmic Ray Conference, Pune (Tata Institute of Fundamental Research, Mumbai, 2005), Vol. 4, p. 271.

[109] E. Andres et al., Astropart. Phys. 13, 1 (2000).

[110] I. A. Belolaptikov et al. (BAIKAL Collaboration), Astropart. Phys. 7, 263 (1997).

[111] H. J. Volk, E. G. Berezhko, and L. T. Ksenofontov, Astron. Astrophys. 433, 229 (2005). 